\title{
Acessibilidade organizacional: barreiras na continuidade do cuidado na atenção primária à saúde
}

Organizational accessibility: barriers in the continuity of health care in primary health care Accesibilidad organizacional: barreras en la continuidad del cuidado en una atención primaria a la salud

\section{Maria Denise Schimith', Maria de Lourdes Denardin Budó'II, Teresinha Heck Weiller ${ }^{\mathrm{III}}$ Lisie Alende Prates ${ }^{I V}$, Laís Antunes Wilhelmv ${ }^{\text {, Gabriela Fávero Albertivi }}$}

Resumo: Objetivo: analisar a acessibilidade organizacional de uma Unidade de Saúde da Família. Método: pesquisa qualitativa na modalidade de estudo de caso, realizada em uma Unidade de Saúde da Família de um município do Rio Grande do Sul, no período de fevereiro a julho de 2012. Resultados: a equipe não se responsabiliza pela recepção dos usuários, interferindo na acessibilidade; as falhas de comunicação entre equipe e usuários, com ausência de acolhimento, prejudicam a resolutividade das necessidades dos usuários; identificou-se que o absenteísmo está relacionado à falta de escuta e singularização; os trabalhadores não se comprometem com a condução de casos. Contudo, linhas de cuidado estão em construção. Conclusões: é necessário qualificar a recepção e a oferta de serviços, fortalecer a organização interna da Unidade e investir na comunicação entre equipe e usuários e com outros pontos de atenção, estabelecendo fluxos assistenciais e redes articuladas.

DESCRITORES: Continuidade da assistência ao paciente; Sistema Único de Saúde; Assistência à saúde; Atenção primária à saúde

Abstract: Objective to analyze accessibility to a Family Health Center. Method: qualitative research in the form of case study, carried out in a Family Health Center from a township in the State of Rio Grande do Sul, from February to July 2012. Results: the team does not take responsibility for the users' reception, which interferes in accessibility; miscommunication between staff and users, and the fact that professionals do not welcome the users undermines the staff's ability to solve the users' needs; it was identified that absenteeism was related to a lack of

\footnotetext{
I Enfermeira. Pós-doutora em Enfermagem. Universidade Federal de Santa Maria. Santa Maria. Rio Grande do Sul. Brasil. ma.denise2011@gmail.com. ORCID 0000-0002-4867-4990

II Enfermeira. Doutora em Enfermagem. Professora aposentada da Universidade Federal de Santa Maria. Santa Maria. Rio Grande do Sul. Brasil lourdesdenardin@gmail.com ORCID: 0000-0002-2539-0813

III Enfermeira. Doutora em Enfermagem. Universidade Federal de Santa Maria. Santa Maria. Rio Grande do Sul. Brasil. weiller2@hotmail.com ORCID 0000-0003-2531-0155

IV Enfermeira. Doutora em Enfermagem. Universidade Federal do Pampa/campus Uruguaiana. Uruguaiana. Rio Grande do Sul. Brasil lisiealende@hotmail.com. ORCID 0000-0002-5151-0292

V Enfermeira, Doutorado em Enfermagem, Universidade Federal de Santa Maria. Santa Maria. Rio Grande do Sul. Brasil. laiswilhelm@gmail.com ORCID 0000-0001-6708-821X

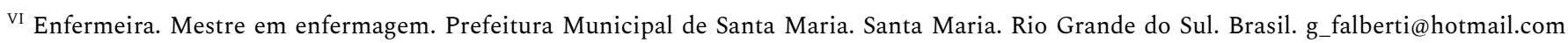
ORCID 0000-0003-1077-7430
} 
Acessibilidade organizacional: barreiras na continuidade do cuidado na atenção primária à saúde | 2

listening and to the absence of personalized care; professionals do not commit to handling the cases. However, lines of care are being created. Conclusions: it is necessary to qualify the reception and service offer, to strengthen the internal organization of the Unit and to invest in the communication between staff and users and with other units, establishing care flows and articulated networks.

KEYWORDS: Continuity of patient care; Unified Health System; Health assistance; Primary health care

Resumen: Objetivo: analizar la accesibilidad organizacional de una Unidad de Salud de la Familia. Método: investigación cualitativa, en la modalidad estudio de casos, realizada en una Unidad de Salud de la Familia, en un municipio de Rio Grande do Sul, en el período de febrero a julio de 2012. Resultados: el equipo no se responsabiliza por la recepción de los usuarios, lo que interfiere en la accesibilidad; hay fallas de comunicación entre el equipo y los usuarios y ausencia de recepción, lo que perjudica la resolución de las necesidades de los usuarios; se identificó que el ausentismo está relacionado con la falta de atención y personalización; el equipo no se compromete con el manejo de los casos. Sin embargo, se están elaborando líneas de atención. Conclusiones: es necesario calificar la recepción y la prestación de servicios, fortalecer la organización interna de la Unidad e invertir en la comunicación entre el equipo y los usuarios y con otros puntos de atención, estableciendo flujos asistenciales y redes articuladas.

DESCRIPTORES: Continuidad de la atención al paciente; Sistema único de Salud; Prestación de atención a la salud; Atención primaria de la salud

\section{Introdução}

O objeto de estudo deste artigo refere-se à acessibilidade organizacional na Atenção Primária à Saúde (APS). "A acessibilidade possibilita que as pessoas cheguem aos serviços, ou seja, este é um aspecto da estrutura de um sistema ou unidade de saúde e este aspecto é necessário para se atingir a atenção ao primeiro contato e a continuidade do cuidado”. 1:225

No Brasil, o Sistema Único de Saúde (SUS) estabelece como um dos seus objetivos, a assistência às pessoas por meio da promoção e recuperação de saúde, prevenção de doença, na conformação de Redes de Atenção à Saúde (RAS). As RAS são propostas dos Sistemas Integrados de Saúde no mundo todo. Definem-se "como arranjos organizativos de ações e serviços de saúde, de diferentes densidades tecnológicas que, integradas por meio de sistemas de apoio técnico, logístico e de gestão, buscam garantir a integralidade do cuidado”. Têm como objetivo integrar as ações e serviços de saúde, de forma "contínua, integral, de qualidade, responsável e humanizada”, além de melhorar o desempenho do SUS, em termos de acesso, equidade, eficácia e eficiência. ${ }^{2: 4}$ 
3 | Schimith MD, Budó MLD, Weiller TH, Prestes LA, Wilhelm LA, Alberti GF

A APS como a porta de entrada preferencial, tem atribuições como o acompanhamento e a organização do funcionamento dos serviços e fluxos de encaminhamentos dos usuários, nos diferentes pontos de atenção das RAS. Para tanto, cabe à APS a responsabilização pela condução dos cuidados aos usuários nos demais pontos da RAS, a partir de uma relação horizontalizada, contínua e integrada, com o objetivo de produzir estratégias de planejamento e gestão compartilhadas da atenção integral, na qual o acesso precisa ser em local e tempo oportunos, com equidade. ${ }^{3}$

Nesse sentido, o acesso aos serviços de saúde representa uma condição necessária para a continuidade do cuidado, e pressupõe a possibilidade de acompanhar, ao longo do tempo, o indivíduo em suas necessidades. Para tanto, são necessários profissionais preparados para o trabalho de cuidados primários, para que seja possível efetivar com qualidade e continuidade o acesso aos serviços e ao cuidado integral. ${ }^{4}$

As barreiras de acesso universal à saúde têm sido identificadas em pesquisas com diversos grupos. Para gestores e profissionais da saúde são: a expansão e o fortalecimento da APS; falta de identificação das principais necessidades; recursos materiais, humanos e tecnológicos deficitários; serviços com infraestrutura precária; baixa resolutividade e ausência de políticas públicas intersetoriais na APS. ${ }^{5}$ Por outro lado, estudo demonstrou que, dentre as dimensões mais valorizadas pelos usuários na APS, está o acesso e a disponibilidade do serviço de saúde; organização do processo de trabalho; relacionamento com os profissionais de saúde; longitudinalidade da atenção à saúde; vínculo entre profissionais e usuários e coordenação do cuidado. $^{6}$

Com isso, justificou-se a presente pesquisa ao lançar luz sobre a organização do trabalho, a partir da perspectiva e da vivência de trabalhadores e usuários de uma Unidade de Saúde da Família (USF), acerca da dimensão da acessibilidade, com a seguinte questão de pesquisa: como a acessibilidade organizacional de uma USF interfere na continuidade do cuidado na RAS? 
Acessibilidade organizacional: barreiras na continuidade do cuidado na atenção primária à saúde $\mid 4$

Teve-se como objetivo analisar a acessibilidade organizacional de uma Unidade de Saúde da Família.

\section{Método}

Tratou-se de uma pesquisa qualitativa, na modalidade de estudo de caso $^{7}$ em que foram contemplados os seguintes componentes essenciais: questões do estudo; proposições; unidade de análise; coerência que articula os dados às proposições e critérios para a interpretação dos dados. A unidade de análise, ${ }^{7}$ ou o "caso”, está relacionado à questão da pesquisa, uma USF.

O cenário do estudo é um município que se localiza no Rio Grande do Sul e é considerado de médio porte. Possui 31 Unidades Básicas de Saúde, serviços especializados, um hospital geral, uma Unidade de Pronto Atendimento, Serviço de Atendimento Móvel de Urgência e um hospital universitário.

A proposta de Estratégia Saúde da Família (ESF) foi implantada em 2003, com 16 equipes. Nas USF atuam equipes mínimas, sendo que cinco dessas unidades possuem equipes de saúde bucal. A unidade de análise é uma USF com duas equipes básicas e equipe de saúde bucal. Para preservar o anonimato da equipe pesquisada, o local da pesquisa não foi revelado, mas ele estabelece o limite espacial do caso. ${ }^{7}$

O limite temporal para a coleta de dados foi de fevereiro a julho de 2012. A coleta de dados seguiu os princípios essenciais de utilização de múltiplas fontes de evidência; a criação de um banco de dados e a manutenção de uma conexão entre estas evidências. ${ }^{7}$

Os participantes da pesquisa foram os trabalhadores de saúde e os usuários da USF. Foram realizadas entrevistas com os trabalhadores e usuários, observação e buscas em documentos.

O critério de inclusão para os trabalhadores foi ser trabalhador ativo da USF durante o período da coleta de dados e para os usuários, que estes tivessem recebido encaminhamento a outro ponto da RAS. Os participantes entrevistados foram duas enfermeiras, duas técnicas de 
5 | Schimith MD, Budó MLD, Weiller TH, Prestes LA, Wilhelm LA, Alberti GF

enfermagem, uma médica, uma odontóloga, uma auxiliar de consultório dentário, um auxiliar de serviços gerais, o coordenador da USF, quatro agentes comunitárias de saúde (ACS) e seis usuários, totalizando 19 entrevistas.

A observação foi do tipo participante, não estruturada e direta, ${ }^{8}$ em dias e turnos alternados, totalizando 76h25min. O diário de campo foi realizado com uma descrição minuciosa, detalhada e densa do objeto de estudo e que auxiliou na análise, pois é fonte rica de informação, não sendo possível captar com outras técnicas de coleta de dados. ${ }^{9}$

Para aumentar a confiabilidade dos dados observados e evitar a parcialidade do pesquisador, $^{7}$ a pesquisa contou-se com duas observadoras auxiliares que se revezaram no acompanhamento das observações. Os diários de campo foram elaborados pela pesquisadora e pelas auxiliares sendo, posteriormente, confrontados e discutidos para garantir maior fidedignidade à observação. A documentação analisada consistiu em cadernos de atas e relatórios da equipe, relatórios de gestão da Secretaria Municipal de Saúde (SMS), agendas dos encaminhamentos e ofícios recebidos.

A elaboração do corpus da pesquisa foi definida como estratégia analítica. Os dados da pesquisa foram revisitados até permitirem a identificação de marcadores importantes para orientar a análise e evitar interpretações a priori.7

As questões éticas foram asseguradas e consideradas, conforme a Resolução nº 196/96, a qual vigorava na época de realização da pesquisa. O projeto foi aprovado sob o protocolo $\mathrm{n}^{\circ}$ 1939/11, em 09 de dezembro de 2011. A participação na pesquisa se deu a partir da aceitação dos participantes, firmada por meio da assinatura do Termo de Consentimento Livre e Esclarecido. O anonimato dos participantes foi viabilizado, mediante a utilização das letras iniciais das funções exercidas pelos trabalhadores, seguidas de representação numérica.

\section{Resultados}


Para apresentar os resultados, optou-se por dividi-los em subtítulos. Assim, estão dispostos como: organização da recepção na USF; falhas na comunicação entre equipe e usuários; absenteísmo e a implicação dos trabalhadores da USF na continuidade do cuidado.

Entre os aspectos da acessibilidade que foram encontrados, a organização da recepção dos usuários na USF foi um obstáculo para a continuidade do cuidado e acesso à RAS. A recepção dos usuários na USF era exercida por dois trabalhadores com desvio de função, uma vez que seus contratos eram como serviços gerais. Na ausência destes, enfermeiras, ACS e técnicas de enfermagem acumulavam esta função. O processo de agendamento dos encaminhamentos aos serviços disponíveis na RAS estava centralizado na trabalhadora da recepção. A equipe não se responsabilizava por ele.

Uma senhora chega ao balcão e entrega um encaminhamento para oftalmologista para Enfa. 1, que anota no verso os dados necessários. A senhora pergunta: para onde que vai ser marcado? Na clínica? Eu quero trocar de médico. Enfa. 1: isso eu não sei, a senhora fala com a recepcionista. [...] a senhora fica com o encaminhamento e vem aqui na segunda falar com ela (recepcionista) para ver se pode. Senhora: e eu queria ver o da minha netinha, faz um tempão que foi pedido e não marcaram ainda, ela tem que quase encostar o rostinho no caderno para poder ler (faz o gesto). Enfa. 1: vê com ela também. (Diário de campo, 19/04/12)

No turno da tarde, quem ficava com essa responsabilidade era o servidor da limpeza que havia assumido por decisão da recepcionista, sem treinamento ou preocupação da equipe.

[...] eu fico ali, não que eu goste, porque não é o meu setor ali. O meu setor é fazer limpeza aqui, mas é para não atrasar o serviço, a pessoa pode precisar de alguma coisa e não ter ninguém para atender ali. (Entrevista com o servidor da limpeza)

Os usuários passam pela experiência de encaminhamentos perdidos ou de não receberem a informação correta sobre os atendimentos nos demais pontos das RAS.

(Reunião de equipe) ACS4: [...] eu oriento a pessoa, disseram que o atendimento não fechava ao meio-dia. Veio a pessoa aqui e disseram: quem está orientando assim? Tem informação desencontrada. 
Coordenador: eu acho que temos que falar as mesmas coisas. (Diário de campo, 29/02/12)

A comunicação entre equipe e usuários foi elencada como obstáculo à acessibilidade. O desencontro de informação gerava peregrinação ao usuário. A equipe não conhecia os fluxos da RAS e justificava a divergência nas informações fornecidas à população, devido às mudanças no fluxo dos serviços ocorridas recentemente.

(Reunião de equipe) [...] ACS3: não é nem uma nem duas pessoas que me reclamaram, foram várias, mas eu também não sabia. Foram marcar exame na secretaria (SMS) e precisa levar comprovante de residência. Várias pessoas na reunião falaram que também não sabiam. [...] ACS4 conta do seu pai que tinha encaminhamento para hematologia e foi informado pela USF que deveria ir à Secretaria Municipal de Saúde e, na verdade, era no Hospital. A Enfa.1 diz que isso mudou há pouco e que não sabiam [...]. Fala também que falta comunicação entre os serviços. (Diário de campo, $30 / 05 / 12)$

O desencontro de informações foi relacionado à chamada 'demanda excessiva' na USF. A equipe reconheceu que a característica do atendimento era de Pronto-Atendimento (PA) e não de USF gerando, para os usuários, o desafio da acessibilidade conforme referência feita ao tamanho da fila que aguardava para a consulta no início da manhã.

A comunicação entre equipe e usuários também estava relacionada com o fato de a equipe não ter se organizado para realizar acolhimento e a justificativa das enfermeiras era de que precisavam de médicos para legitimar a prática. Além disso, e também inerentes à acessibilidade, foram as restrições quanto ao número de consultas médicas (apenas 15 por turno) e o horário que o usuário deveria chegar à USF para garantir o atendimento.

(Reunião de equipe) Enfa. 1: hoje de manhã aconteceu de novo, não é a primeira vez, hoje veio uma criança com dor de garganta, foi lá no [nome do hospital] e disseram que não caracterizava como um atendimento de Pronto Atendimento, chegou aqui às $10 \mathrm{~h}$ e não tinha mais médico. Tem que orientar o povo para não ir para o [hospital]. (Diário de campo, $02 / 05 / 12)$ 
A ausência do médico, às 10 horas, em uma USF interferiu na utilização do serviço de saúde, pois constituiu-se em uma experiência sem êxito para os usuários. Além da peregrinação dos usuários, o distanciamento da equipe e a falta de acolhimento, os usuários não tinham seu problema resolvido, levando ao afastamento do serviço.

O alto índice de absenteísmo em consultas e exames encaminhados a especialidades em outros pontos da RAS, constituiu uma barreira à continuidade do cuidado nessa USF. A razão disso estava relacionada à falta de escuta e de singularização, além do atraso no agendamento da consulta.

Na recepção, uma senhora fala com a recepcionista: meu exame com o reumatologista? Já marcaram? [...] Recepcionista: mas isso vai para secretaria (SMS) só uma vez por mês. Senhora: eu sei, mas faz muitos meses que já estou esperando. A recepcionista pega o envelope de retorno para o reumatologista e não encontra o pedido da senhora. A recepcionista encontra o pedido na pasta com identificação de "perdidos" e diz: está aqui, foi agendado para 14 de maio, liguei e ninguém atendeu, vou colocar de volta para marcar de novo. Senhora: coloca o telefone do outro filho, ele atende. (Diário de campo, 19/06/12)

A recepcionista relatou que muitas pessoas eram avisadas sobre as consultas agendadas e não buscavam o encaminhamento. Outras, ela não conseguia avisar. Na pasta nomeada “perdidos” estavam vários destes encaminhamentos, alguns sem nenhuma anotação.

Pesquisadora: há alguns que, possivelmente, foram recebidos e não agendados. $A$ recepcionista sacode os ombros, com expressão de descompromisso. A pesquisadora conta o número de pedidos "perdidos". Recepcionista: está vendo, esse povo não tem jeito, depois vêm reclamar que não são atendidos, mas olha quantos que não vão às consultas, a maioria foi avisada, são pedidos recentes. Pesquisadora: nem todos são recentes e nem todos foram avisados. (Diário de campo, 19/06/12)

Nesse caso, o absenteísmo estava relacionado com a forma como a recepcionista se relacionava com os usuários. Nessa observação, a culpa pelas ausências às consultas e exames 
9 | Schimith MD, Budó MLD, Weiller TH, Prestes LA, Wilhelm LA, Alberti GF

foi transferida a eles. Algumas condutas da recepção evidenciaram o desrespeito ao usuário e a falta de singularização de cada caso.

[...] A recepcionista recebe um encaminhamento de um senhor, diz que ligará quando for marcado. O senhor diz que tem que ligar depois das $16 \mathrm{~h}$. Recepcionista: está bem. A pesquisadora lembra a recepcionista que a USF fecha às 16h. Senhor: então, liga perto do meio-dia. Recepcionista: está bem? O senhor sai e ela fala: eu lá quero saber que horas tem gente em casa, eu ligo, se está, está, se não, azar. Coloca o formulário no envelope, sem anotar nada. (Diário de campo, 27/04/12)

A falta de singularização e a ausência de liberdade de escolha do usuário implicaram na dificuldade de acessar a RAS. A recepção de uma USF que pretenda garantir o menor tempo de acesso a outro serviço, precisa ser supervisionada pela equipe sob pena de não atingir seus objetivos.

Foi possível também identificar ausência de implicação dos trabalhadores da USF na continuidade do cuidado. Diante disso, como primeiro elemento, estava a forma como as enfermeiras priorizavam a utilização do prontuário de família.

(Reunião de equipe) $A$ Enfa. 2 fala que os pacientes estão chegando sem receita e sem prontuário, que fica difícil para o médico prescrever. Solicita que as ACS orientem que os pacientes devem trazer a última receita ou o número do prontuário. Enfa. 1: um paciente chegou com Pressão Arterial $160 / 100 \mathrm{mmHg}$ e não sabia que medicação tomava, não sabia o número do prontuário, não sabia nada, assim fica difícil. A pesquisadora sugere o retorno da planilha Excel com o cadastro [...]. ACS 1 diz que não acredita que as colegas não orientam. [..] A Enfa. 2 fala que não é só orientar e, sim, conscientizar. (Diário de campo, 23/05/12)

A falta de priorização na atualização do cadastro da população de abrangência afetou a acessibilidade organizacional, o que interferiu na continuidade da atenção. A equipe não compartilhava da responsabilidade na continuidade do cuidado, tampouco era propositiva em pensar alternativas para que o processo de trabalho facilitasse a atualização cadastral dos usuários. 
A continuidade do cuidado também ficou prejudicada com a falta de implicação das enfermeiras, sentida pelas ACS. Questionada sobre a inserção da USF na RAS, a ACS1 mencionou situações em que não havia acolhimento e corresponsabilização da equipe pelos casos.

[...] sabe aquelas redes de pescar? Quando passa um peixe bem grande, como é que ela fica? Furada (risos), assim que eu enxergo, porque não tem resolução dos casos. [...] fica só na primeira telinha da rede. Tu fazes a visita e o paciente precisa de mais alguma coisa, e não desenvolve o caso [...] mas se tiver uma ponta da rede que seja bem tramadinha, ela funciona [...] Se eu tivesse um enfermeiro de perfil, se eu tivesse um técnico de perfil, um médico de perfil, essa parte da rede funcionaria [...] Daí tu dizes assim: já teve isso? Sim, teve sim, existiu sim essa rede [...] tinha resolução, tu tinhas prazer em trazer um caso, porque ele era resolvido a tempo de não estourar no [hospital] de alta complexidade. A prevenção, a orientação e a informação tu fazes, mas aquela parte fica falha, bem falha. (Entrevista com ACS1)

A simbologia, "rede de pescar furada”, usada para descrever a RAS que era vivenciada pela ACS, revelou a ausência de condução dos casos. Muitas vezes, as ACS expõem os casos para a equipe e não percebem a implicação necessária para que a resolutividade seja atingida.

No entanto, durante o período de coleta, observou-se o nascimento da construção de linhas de cuidado, a partir do protagonismo da Residência Multiprofissional Integrada do Sistema Público de Saúde (RMPISPS).

A Enfa.1 conta que a enfermeira da clínica cirúrgica do Hospital (projeto transferência do cuidado da residência multiprofissional) telefonou e passou o caso de uma senhora que foi encaminhada pela USF e passou por uma amputação no hálux, solicitando curativo domiciliar. (Diário de campo, 16/05/12)

[...] agora que o hospital está ligando para agendar as puericulturas e as consultas do puerpério. As gestantes vão para lá, no Centro Obstétrico, fazem o parto lá e vão para a maternidade, de lá ligam para cá para agendar o retorno. Pesquisadora: agendar com vocês, antes de dar alta? Enfa. 2: 
isso, antes de dar alta [...] não estava acontecendo, acho que a Residência que está fazendo. (Entrevista com a Enfa. 2)

Movimentos como esse reforçam que a acessibilidade na USF e a continuidade do cuidado são possíveis e que estão ligadas às relações estabelecidas dentro do serviço e com outros pontos da RAS. A RMPISPS destaca-se como protagonista na construção do SUS.

\section{Discussão}

A acessibilidade organizacional pode ocorrer na entrada ou no interior da USF. ${ }^{10} \mathrm{Na}$ recepção da USF estudada, havia ausência de padronização no atendimento, condutas solitárias e não interativas entre trabalhadores que geravam dificuldade de acessibilidade para os usuários. Esta função deveria ser uma preocupação constante das equipes e da gestão, pois a questão de "como" a população estava sendo recebida nas portas dos serviços pôde determinar a confiança e a acessibilidade aos demais pontos da RAS.

Os resultados corroboraram com a pesquisa realizada nos municípios da região norte do Estado de São Paulo. No estudo, foi identificada a fragilidade na posição da APS, tanto como ponto regular de cuidado, quanto porta de entrada preferencial na RAS. ${ }^{3}$

Nesse sentido, destaca-se a necessidade de os serviços de saúde, principalmente na APS, aderirem ao modelo centrado nas necessidades das pessoas, pois há comprovação de que é mais eficaz e mais efetivo. O fortalecimento da APS é uma das bandeiras da Organização Mundial da Saúde (OMS) que, desde a metade do século XX, propõe atingir a meta de saúde para todos. ${ }^{11}$

No interior da USF, o usuário não era singularizado em relação às suas necessidades, sendo que os formulários de encaminhamentos eram perdidos, o que ocasionava demora no agendamento, atrasos nas consultas e aumento no tempo de espera. O recebimento da demanda estava centralizado em funcionários com desvio de função; a equipe não se apropriava dessas atribuições e havia desencontros de informações sobre os serviços e suas funções, em virtude do 
Acessibilidade organizacional: barreiras na continuidade do cuidado na atenção primária à saúde | 12

desconhecimento dos fluxos da RAS. Todos esses aspectos foram considerados barreiras à acessibilidade. $^{10}$

Um estudo corroborou com a presente pesquisa em que informantes da equipe multiprofissional identificaram como principais barreiras ao acesso universal à saúde, dentre outras, a ausência de reconhecimento de demandas prioritárias e baixa resolutividade em ações de saúde. No entanto, diversamente à presente pesquisa, a enfermagem foi destacada com um olhar aprofundado sobre a realidade em que atuava, contribuindo para a equidade na cobertura e acesso universal. ${ }^{5}$

Verificou-se que os registros nos prontuários não eram priorizados e a responsabilização era repassada para as ACS e para a população. Isso constituiu barreiras relacionadas à acessibilidade organizacional, compreendida como "obstáculos que se originam nos modos de organização dos recursos de assistência à saúde”. 10:7

Uma pesquisa também revelou que a dimensão sócio-organizacional ainda é uma barreira no acesso à APS no Estado de Pernambuco e que o acolhimento e a coordenação do cuidado eram marcadores presentes da APS e relevantes para a acessibilidade. ${ }^{12}$ Outro estudo identificou que a prevalência de fonte regular de serviços foi baixa, tanto em usuários afiliados à UBS quanto ESF, indicando que isso implicava no estreitamento do vínculo e proporcionava o acompanhamento dos pacientes. ${ }^{13}$

É importante para a APS e para RAS que a população seja adscrita, ou seja, vinculada a um serviço, pois um dos pilares do modelo integrado de saúde é a base populacional. O conhecimento da população usuária é elemento-chave para eliminar a organização do serviço baseado na oferta, que é característico de sistemas fragmentados. ${ }^{14}$

Reforça-se, assim, a implicação necessária dos trabalhadores da USF para garantir a continuidade do cuidado. Espera-se que estes realizem a conexão com os outros pontos da RAS, conduzindo o cuidado necessário para cada caso e possibilitando sua resolução. 
As linhas de cuidado implementadas pela RMPISPS representam alternativas micropolíticas na construção das RAS no SUS. Esta prática destaca-se por não existir um único caminho para a transformação social, pois se convive com uma sociedade que é, ao mesmo tempo, autoritária e libertária. Assim, como são inúmeras as formas de opressão e dominação, também são várias as formas de resistência e os agentes protagonistas. ${ }^{15}$

A postergação de resolubilidade dos problemas de saúde trazidos pelas ACS também revelou a simplificação da complexidade dos casos na perspectiva da equipe, interferindo na continuidade da atenção e no acesso ao serviço de saúde. Quando a equipe não se mobilizava para resolver o problema, o caso era encaminhado ao serviço de alta densidade tecnológica.

Percebeu-se que havia necessidade de a equipe desenvolver um trabalho em colaboração e que para o cuidado colaborativo acontecer é fundamental que a habilidade social e profissional dos gestores do cuidado. ${ }^{16}$

A equipe solicitava às ACS que orientassem os usuários a não procurar o PA para o tratamento de agravos passíveis de intervenção na USF. Assim, a questão de fundo parece ser a construção de vínculo como dispositivo que possibilita a adesão à terapêutica e/ou a continuidade do tratamento, evitando consultas e internações desnecessárias. ${ }^{17}$

A preferência do usuário em procurar o atendimento que lhe proporcionasse resolubilidade imediata, como o PA, pode estar relacionada à falta de organização da equipe para atender a demanda espontânea dos usuários. Portanto, não se caracterizava como porta de entrada, tampouco permitia a longitudinalidade do cuidado, corroborando com outro estudo. ${ }^{13}$

As barreiras na acessibilidade permitiram afirmar que, tanto os princípios da universalidade quanto da integralidade do SUS, não estavam assegurados. Nesse contexto, a humanização também ficou comprometida, pois ações normativas e burocráticas não eram suficientes para garantir práticas de cuidado e processos acolhedores que intentassem melhorias na acessibilidade aos serviços de saúde. ${ }^{18-19}$ 
Para agir na complexidade aqui apresentada, é necessário fugir da armadilha de justificar o que existe e apostar na recuperação da esperança. A esperança reside na "possibilidade de criar campos de experimentação social onde seja possível resistir localmente às evidências da inevitabilidade, promovendo com êxito alternativas que parecem utópicas em todos os tempos e lugares”. ${ }^{20: 36}$

Uma possível limitação deste estudo foi a opção metodológica pelo estudo de caso ter sido realizado apenas em uma USF, revelando aspectos específicos da organização desta. No entanto, foi possível a compreensão do fenômeno da acessibilidade organizacional neste espaço, possibilitando a generalização na perspectiva teórica, como evidenciado na comparação a outros estudos.

\section{Conclusões}

Essa pesquisa identificou a forma como os usuários eram recebidos, relacionando a acessibilidade organizacional e a sua movimentação na RAS. O cotidiano da USF estudada apresentou barreiras organizacionais importantes para a acessibilidade, que precisariam ser enfrentadas. Disso depende a reestruturação do modelo de atenção, tornando-o eficaz e eficiente. A recepção do serviço é fundamental num serviço de saúde, pois é a partir dela que se tece a conexão do usuário com o que se oferta no interior da unidade e externo a ela, na RAS. Quando isso não é valorizado e há uma divergência de informações, a recepção dos usuários pode tornar-se fragilizada, sem responsabilização e priorização pela demanda espontânea.

Os obstáculos reais que o usuário enfrenta na busca pelo cuidado contínuo e integral no SUS precisam ser enfrentados, sob pena de colocar a continuidade do cuidado em risco. É necessário ampliar e qualificar a oferta de serviços, fortalecer a organização interna da USF, com vistas a consolidar a ESF como fonte regular do cuidado. Recomenda-se também investir na 
15 | Schimith MD, Budó MLD, Weiller TH, Prestes LA, Wilhelm LA, Alberti GF

comunicação com outros pontos de atenção do SUS, estabelecendo fluxos assistenciais e favorecendo a construção de uma RAS articulada.

\section{Referências}

1. Starfield B. Atenção primária: equilíbrio entre necessidades de saúde, serviços e tecnologia. Brasília (DF): UNESCO, Ministério da Saúde; 2002.

2. Brasil. Ministério da Saúde. Portaria n. 4.279, de 30 de dezembro de 2010. Estabelece diretrizes para a organização da Rede de Atenção à Saúde no âmbito do Sistema Único de Saúde (SUS). Diário Oficial da União, Brasília; 2010 dez 30. Seção 1, p. 89.

3. Bousquat A, Giovanella L, Campos EMS, Almeida PF, Martins CL, Mota PHS et al. Atenção primária à saúde e coordenação do cuidado nas regiões de saúde: perspectiva de gestores e usuários. Ciênc Saúde Colet [Internet]. 2017 [acesso em $2018 \mathrm{dez}$ 18];22(4):1141-54. Disponível em: http://www.scielo.br/pdf/csc/v22n4/1413-8123-csc-22-04-1141.pdf doi: 10.1590/1413-81232017224.28632016

4. Figueira MCS, Silva WP, Silva EM. Integrative literature review: access to primary healthcare services. Rev Bras Enferm [Internet]. 2018 [acesso em 2018, dez 18];71(3):1178-88. Disponível em: http://www.scielo.br/pdf/reben/v71n3/0034-7167-reben-71-03-1178.pdf doi: 10.1590/0034-7167-2017-0441

5. França VH, Modena CM, Confalonieri UEC. Visão multiprofissional sobre as principais barreiras na cobertura e no acesso universal à saúde em territórios de extrema pobreza:

contribuições da enfermagem. Rev Latinoam Enferm [Internet]. 2016 [acesso em 2018 dez 11];24:e2795. Disponível em: http://www.scielo.br/pdf/rlae/v24/pt_0104-1169-rlae-24-02795.pdf doi:10.1590/15188345.1042 .2688

6. Moraes VD, Campos CEA, Brandão AL. Estudo sobre dimensões da avaliação da Estratégia Saúde da Família pela perspectiva do usuário. Physis (Rio J) [Internet]. 2014 [acesso em 2018 dez 11];24(1):127-46. Disponível em: http://www.scielo.br/pdf/physis/v24n1/0103-7331-physis-24-01-00127.pdf doi: 10.1590/S0103-73312014000100008

7. Yin RK. Estudo de caso: planejamento e métodos. 4ª ed. Porto Alegre: Bookman; 2010.

8. Polit DF, Beck CT. Fundamentos de pesquisa em enfermagem: avaliação de evidências para a prática da enfermagem. 9ª ed. Porto Alegre: Artmed; 2018.

9. Tonin L, Lacerda MR, Favero L, Nascimento JD, Zagonel IPS. Diário de campo na pesquisa qualitativa de enfermagem. In: Lacerda MR, Ribeiro RP, Costenaro RGS, organizadores. Metodologia da pesquisa para a enfermagem e saúde: da teoria à prática. v. 2. Porto Alegre: Moriá; 2018. p. 373-98. 
Acessibilidade organizacional: barreiras na continuidade do cuidado na atenção primária à saúde | 16

10. Fekete MC. Estudo da acessibilidade na avaliação dos serviços de saúde. In: Santana JP, Santos I, Fekete MC, Galvão EA, Mandelli MJ, Penna MLF, et al., organizadores. Desenvolvimento gerencial de Unidades Básicas do Sistema Único de Saúde (SUS). Brasília (DF): Organização Pan-Americana da Saúde; 1997. p. 177-84.

11. Organización Mundial de la Salud (OMS). Más sano, más justo, más seguro: la travesía de la salud mundial 2007-2017. Ginebra: Organización Mundial de la Salud; 2017.

12. Albuquerque MSV, Lyra TM, Farias SF, Mendes MFM, Martelli PJL. Acessibilidade aos serviços de saúde: uma análise a partir da Atenção Básica em Pernambuco. Saúde Debate [Internet]. 2014 [acesso em 2015 nov 12];38(esp):182-94. Disponível em: http://www.scielo.br/pdf/sdeb/v38nspe/0103-1104-sdeb-38spe-0182.pdf doi: 10.5935/0103-1104.2014S014

13. Lima-Costa MF, Turci MA, Macinko J. Estratégia Saúde da Família em comparação a outras fontes de atenção: indicadores de uso e qualidade dos serviços de saúde em Belo Horizonte, Minas Gerais, Brasil. Cad Saúde Pública [Internet]. 2013 [acesso em 2015 nov 21];29(7):1370-80. Disponível em: http://www.scielo.br/pdf/csp/v29n7/11.pdf doi: 10.1590/S0102-311X2013000700011

14. Brasil. Conselho Nacional de Secretários de Saúde. A Atenção Primária e as Redes de Atenção à Saúde/Conselho Nacional de Secretários de Saúde. Brasília (DF): CONASS; 2015. 127 p.

15. Santos BS. Para um novo senso comum: a ciência, o direito e a política na transição paradigmática. $7^{\text {a }}$ ed. São Paulo: Cortez; 2009.

16. Overbeck G, Davidsen AS, Kousgaard MB. Enablers and barriers to implementing collaborative care for anxiety and depression: a systematic qualitative review. Implement Sci [Internet]. 2016 [acesso em 2018 dez 11];11:165. Disponível em:

https:/www.ncbi.nlm.nih.gov/pmc/articles/PMC5192575/pdf/13012_2016_Article_519.pdf doi: 10.1186/s13012-016-0519-y

17. Barbosa MIS, Bosi MLM. Vínculo: um conceito problemático no campo da Saúde Coletiva. Physis (Rio J) [Internet]. 2017 [acesso em $2018 \mathrm{dez}$ 18];27(4):1003-22. Disponível em: http://www.scielo.br/pdf/physis/v27n4/0103-7331-physis-27-04-01003.pdf doi: 10.1590/s010373312017000400008

18. Brasil. Ministério da Saúde. Departamento de Atenção Básica. Acolhimento à demanda espontânea. Brasília (DF): Ministério da Saúde; 2013.

19. Ribeiro JP, Mamed SN, Souza MR, Souza MM, Rosso CFW. Acessibilidade aos serviços de saúde na Atenção Básica do Estado de Goiás. Rev Eletrônica Enferm [Internet]. 2015 [acesso em 2016 set 04];17(3):1-11. Disponível em: https://www.fen.ufg.br/revista/v17/n3/pdf/v17n3a04.pdf doi:10.5216/ree.v17i3.29436 
17 | Schimith MD, Budó MLD, Weiller TH, Prestes LA, Wilhelm LA, Alberti GF

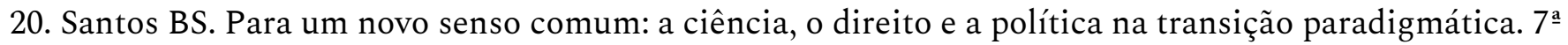
ed. São Paulo: Cortez; 2009.

\section{Autor correspondente}

E-mail: ma.denise2011@gmail.com

Endereço: Rua João Goulart, 519. Camobi. Santa Maria, RS

CEP: $97105-220$

\section{Contribuições de Autoria}

1 - Maria Denise Schimith

Concepção e planejamento do projeto de pesquisa; obtenção dos dados, análise e interpretação dos dados; redação e revisão crítica do manuscrito.

2 - Maria de Lourdes Denardin Budó

Concepção e planejamento do projeto de pesquisa, interpretação dos dados e revisão crítica do manuscrito.

3 - Teresinha Heck Weiller

Interpretação dos dados, redação e revisão crítica do manuscrito.

4 - Lisie Alende Prestes

Interpretação dos dados, redação e revisão crítica do manuscrito.

5 - Laís Antunes Wilhelm

Interpretação dos dados, redação e revisão crítica do manuscrito.

6 -Gabriela Fávero Alberti

Interpretação dos dados, redação e revisão crítica do manuscrito.

\section{Como citar este artigo}

Schimith MD, Budó MLD, Weiller TH, Prestes LA, Wilhelm LA, Alberti GF. Acessibilidade organizacional: barreiras na continuidade do cuidado na atenção primária à saúde. Rev. Enferm. UFSM. 2019 [Acesso em: 2019 jun 15];vol ex:p1-p18. DOI:https://doi.org/10.5902/2179769228053 